\title{
Article
}

\section{Selective extraction of proteins and other macromolecules from biological samples using molecular imprinted polymers}

Stevenson, D, EL-Sharif, H and Reddy, Subrayal M

Available at http://clok.uclan.ac.uk/15732/

Stevenson, D, EL-Sharif, $H$ and Reddy, Subrayal M ORCID: 0000-0002-7362$184 X$ (2016) Selective extraction of proteins and other macromolecules from biological samples using molecular imprinted polymers. Bioanalysis, 8 (21). pp. 2255-2263. ISSN 1757-6180

It is advisable to refer to the publisher's version if you intend to cite from the work. http://dx.doi.org/10.4155/bio-2016-0209

For more information about UCLan's research in this area go to http://www.uclan.ac.uk/researchgroups/ and search for <name of research Group>.

For information about Research generally at UCLan please go to http://www.uclan.ac.uk/research/

All outputs in CLoK are protected by Intellectual Property Rights law, including Copyright law. Copyright, IPR and Moral Rights for the works on this site are retained by the individual authors and/or other copyright owners. Terms and conditions for use of this material are defined in the policies page. 
1 Selective extraction of proteins and other macromolecules from

2 biological samples using molecular imprinted polymers

3 Derek Stevenson ${ }^{*}{ }^{1}$, Hazim F EL-Sharif ${ }^{1}$ \& Subrayal M Reddy ${ }^{2}$

$4 \quad{ }^{1}$ Chemistry Department, University of

5 Surrey, Guildford, Surrey, GU2 7XH, UK

$6 \quad{ }^{2}$ Chemistry Division, University of

7 Central Lancashire, Preston, Lancashire,

8 PR1 2HE, UK

9 *Author for correspondence:

10 d.stevenson@surrey.ac.uk 


\section{Background}

13 The determination of drugs, metabolites and biomarkers in biological samples

14 continues to present one of the most difficult challenges to analytical scientists.

15 Matrices such as plasma, serum, blood, urine or tissues for example, usually

16 contain the analyte(s) of interest at low concentration in the presence of many

17 other components which may interfere directly or indirectly with the accurate

18 determination of species and concentration. Historically, the most common

19 methods have involved some form of extraction or isolation such as liquid-liquid

20 extraction (LLE), solid phase extraction (SPE) or protein precipitation. For a

21 recent review of sample preparation methods for bioanalysis, see [1]. This

22 includes comments on costs, automation, and miniaturisation with an overall

23 focus on productivity.

25 Accurate quantitative measurement over the last 40 years has traditionally been

26 carried out by chromatography, mainly high performance liquid chromatography

27 (HPLC) and occasionally gas chromatography (GC). Although a range of

28 detectors has been available for both, most typically, HPLC used ultraviolet (UV)

29 and GC used flame ionisation and then both have used mass spectrometry (MS).

30 Sample preparation has been usually by a variant of LLE, SPE or protein

31 precipitation [2]. As the need for greater sensitivity has been a constant

32 challenge, sophisticated and more selective methods of sample preparation have

33 been explored. One of the most attractive of these has been the use of

34 immobilised antibodies [3] to selectively extract drugs and metabolites in a typical 
35 SPE format. Many examples have been published but the approach has been

36 limited by a number of factors such as cost and uncertainty of antibody

37 production as well as stability of the antibodies. Significant developments

38 overcoming the coupling of MS to HPLC and its subsequent widespread use has

39 meant that the demands on sample preparation have been reduced. As drugs

40 and metabolites are typically small molecular mass organic compounds greater

41 selectivity and sensitivity could be achieved by the end step measuring

42 technique, and there has indeed been wide uptake of this technology especially

43 in the pharmaceutical industry.

\section{Molecularly imprinted polymers}

46 Nevertheless, within the bioanalytical community the interest in the advantages

47 offered by selective extraction have remained. Molecularly imprinted polymers

48 (MIPs) have been suggested as an alternative to immobilised antibodies in a

49 number of areas including bioanalysis [4] as these are potentially much cheaper

50 to synthesise and more stable than biological antibodies. MIPs have been the

51 subject of numerous reviews $[5,6]$ so the basic principles will only be

52 summarised here. Briefly, the preparation involves a reaction mixture containing

53 the analyte (the so-called template molecule), a functional monomer, a cross-

54 linking agent and an initiator in a suitable solvent. The MIP is formed around the

55 template. The template is subsequently removed leaving cavities that can

56 selectively rebind the template. The aim is to create a reagent (MIP) that can

57 selectively bind the analyte, in a similar way to an antibody. Immobilized 
58 antibodies can be very specific but they are inherently quite fragile molecules,

59 particularly when exposed to organic solvents, $\mathrm{pH}$ values of more than 2-3 units

60 from neutral and/or heat. They can also be quite time-consuming to produce, in

61 many cases requiring repeated dosing to animals, with no certainty that useful

62 antibodies will eventually be obtained. In contrast, MIPs are produced rapidly in

63 the chemistry laboratory and use well-established synthetic routes which lead to

64 comparatively lower production costs. They are more stable over a wider $\mathrm{pH}$

65 range and can be used with a broader range of solvents. This potentially also

66 offers the advantage that they could be re-usable, further lowering the costs.

67

68 Many papers and reviews have been written on the optimisation of conditions,

69 different methods of characterisation and different uses of MIPs [7-9]. In many

70 cases, the MIPs will only perform their selective capture if they are in the solvent

71 used for their preparation. The importance of buffer conditioning has been

72 emphasised [9]. For example, MIPs have been proposed as offering advantages

73 as columns for HPLC, SPE [2, 3, 10, 11], capillary electrophoresis [12] and

74 electrochromatography, replacing antibodies in enzyme-linked immunosorbent

75 assay (ELISA) tests [13], artificial enzymes or receptors, recognition elements

76 within sensors [14], selective drug delivery, catalysts and to aid crystallization

77 [15]. The area of SPE has attracted most attention and new approaches are still

78 being reported in this area. The development of nanoparticles has led to

79 molecular imprinting onto the surface of magnetic nanoparticles [16] followed by

80 solid phase microextraction (SPME) or ultrasonic assisted SPME [17-19] and 
81 matrix dispersant SPME [20, 21]. MIPs which are integrated with magnetic

82 nanoparticles offers the added advantage of a simple separation using a magnet

83 following the selective template (analyte) binding/extraction step. Ding et al. 2014

84 [22] has written a recent review on surface imprinting technologies for nano-

85 MIPs. This described both small and large molecule templates in two different

86 sections. Examples of biomacromolecules that have been imprinted include

87 lysozyme, bovine haemoglobin, human haemoglobin, amylase and bovine serum

88 albumin (BSA) as well as virus particles.

90 The preponderance of reviews on the use of MIPs for separation science has led

91 to a review of reviews [23]. Nonetheless the use of commercially available MIPs

92 using validated methods for bioanalysis is not considered commonplace. Li et al.

932014 [24] has written an extensive review on macromolecules concentrating on

94 proteins, carbohydrates, DNA, viruses and cells. The review contrasts the

95 development of small molecular mass versus macromolecule templates.

96 Progress with the latter has been slower and unremarkable. Several commercial

97 companies are producing MIPs for SPE mainly for small molecular mass analytes

98 such as drugs and pesticides. These however are not commonplace.

99

100 Many of the applications published in scientific literature consider only the 101 comparison of a MIP with a non-imprinted polymer (NIP), along with comparisons 102 of a very small number of other related compounds as evidence of a MIP effect.

103 Studies looking at the rebinding of the analyte to the MIP compared to a NIP are 
104 commonplace. In many applications the MIPs will often only work satisfactorily 105 when the rebinding is carried out in the solvent in which the MIP was 106 synthesised, typically organic solvents. This is a considerable drawback when the 107 need is to extract from aqueous biological fluids such as plasma, serum, urine, 108 tissue extracts and faeces. It is also unsuitable for most macromolecules of 109 biological interest as they are not stable in organic solvents. Biologicals (greater 110 than $1000 \mathrm{Da})$ are metastable and can undergo intra-molecularly-induced 111 changes in conformation depending on their chemical environment. They

112 therefore need to be exposed to less harsh polymerisation conditions compared 113 with the imprinting of small and robust molecules (less than $700 \mathrm{Da}$ ) the latter 114 inherently possessing less degrees of freedom in molecular arrangement. MIP 115 preparations for biologicals have therefore focused on the use of water116 compatible polymers, namely hydrogels based on using acrylamide (AAm) as a 117 functional monomer [25-27] and the repertoire extended more recently using a 118 combination of acrylo-based functional monomers to polymerise in the presence 119 of a second (more biocompatible) polymer including polyethylene glycol (PEG) 120 and chitosan [28]. Chitosan is a derivative of chitin (extracted from crustacean 121 species), and is produced by deacetylation of chitin under alkaline conditions. At 122 around physiological $\mathrm{pH}$ and below, chitosan is positively charged. Thus in 123 addition to the generally accepted hydrogen bonding interactions and cavity fit 124 offered by MIPs, the presence of positive charge offers an additional 125 (electrostatic) anchor for the imprinting of proteins. 


\section{MIPs for extraction/enrichment of macromolecules}

128 One area of growing interest in bioanalysis has been in the preparation of MIPs

129 to peptides, proteins or other large biomolecules $[29,30]$. The changing nature of

130 drug development suggests that macromolecules are increasingly being

131 proposed as new therapeutic agents or indeed as biomarkers for a range of

132 diseases. Novel approaches for their reliable accurate measurement is thus of

133 growing interest. In many cases the macromolecules will be present in biological

134 fluids at low concentrations so the application of MIPs for selective extraction to 135 allow pre-concentration and clean-up is a very attractive approach. The 136 development of such MIPs using protein templates was reviewed [31]. The latter 137 review was focused on sensors but the methods used to prepare the MIPs 138 should be a useful guide for their eventual application in selective enrichment or 139 other applications. The review discussed template selection, bulk compared with 140 surface imprinting, the use of whole protein or epitopes, solvent conditions used 141 for imprinting, the choice of monomers and cross-linkers, procedures for template 142 removal as well as the sensor development aspects, Many of the examples of 143 MIPs for proteins use a low degree of cross-linking to give soft hydrogels rather 144 than the highly cross-linked rigid gels used for small molecule imprinting. The 145 advantages of using surface imprinting when preparing protein MIPs has been 146 described in ref [32]. This review included sections on SPE, mainly of small 147 molecular mass analytes. The use of carbon nanofibres, nanodiamonds, 148 fullerenes, carbon nanotubes, graphene and graphene oxide were evaluated by 
149 ref [33] as possible materials for isolation and pre-concentration of proteins and 150 where MIPs can improve selectivity.

152 There have been several reviews of the use of MIPs for SPE. For example, 153 Augusto et al. 2013 [34] considered the merits of immunoaffinity, MIPs, 154 aptamers, carbon nanotubes and other nanomaterials. These give numerous 155 examples of the use of MIPs to extract small molecular mass compounds but 156 generally give few examples of macromolecule extraction. SPE can be carried 157 out in several formats. Examples include a conventional small syringe packed 158 with the MIP, coated fibres, capillaries, surface coated particles, coated stir bars, 159 membranes, magnetic beads and nanoparticles [35]. All have advantages and 160 disadvantages and these were evaluated. Hu et al. 2013 also emphasised that 161 the major obstacles include the difficulty of finding optimised conditions for 162 selective extraction, compatibility with aqueous solutions and the low number of 163 binding sites obtained [35].

165 Schirhagl et al. 2014 [36] reviewed the particular approaches to imprinting large 166 biomolecules and highlighted the advantages of using more flexible polymers 167 than the rigid polymers used for small molecules. The review covered methods of 168 synthesis, template removal, applications using various methods (optical, 169 electrical and mass sensitive) of signal production in sensors, separation science 170 and possibilities in drug discovery. The article concluded that selectivities 
171 obtained for large biomolecules are still not as good as those for small drug like 172 molecules.

174 One interesting approach recently reported was the use of a surface imprinted 175 polymer using myoglobin as the template [37]. The MIP allowed selective capture 176 and release of the target using temperature, rather than the much more 177 widespread use of a change of solvent or $\mathrm{pH}$.

179 The basic principle of using a selective extraction followed by desorption into a 180 chromatograph with an MS detector or other instrumental technique is attractive, 181 as accurate measurement and a high degree of specificity or identification can be 182 achieved. Again the evidence quoted in scientific literature for a MIP effect is 183 often that the macromolecule is extracted with greater recovery from the MIP 184 than the NIP and selectivity to similar molecules in terms of molecular mass, 185 function or isoelectric potential. Conclusive evidence of a molecular imprinting 186 effect has been questioned [38]. Although comparison of MIP to NIP is some 187 evidence of a MIP effect the non-specific binding to the NIP does suggest that 188 further studies such as structural characterization would be helpful. Non-specific 189 binding will prove to be a particular obstacle to widespread acceptance when 190 complex samples such as biofluids are processed. Ultimately, the crucial point is 191 not whether the selective capture is an effect requiring specific interactions at 192 specific points on the polymer; rather, it is whether or not MIP-based selective 
193 extraction provides improvement in the analytical methods developed. This would

194 then need widespread uptake to become completely convincing.

195 This article will review recent examples in the development of the use MIPs for

196 selective extraction or enrichment of proteins and other large biomolecules

197 appropriate to biological samples. A very extensive collection of articles

198 describing the preparation or use of MIPs in all their applications is listed online

199 [39]. The majority of applications of MIPs are in the area of separation science or

200 sensors. The reality that there are few examples of methods based on MIPs for

201 selective extraction of macromolecules suggests something of an unmet need 202 here.

203

204 Examples of extraction/enrichment of macromolecules using MIPs (see 205 also Table 1)

206 Qadar et al. 2014 [40] developed MIPs to the nonapeptide progastrin releasing 207 peptide (ProGRP), a possible biomarker for small cell lung cancer. A range of 208 acrylamide monomers were evaluated in the SPE format with fractions analysed 209 by HPLC-UV. Selectivity was checked against 4 other peptides. In a follow up 210 paper [41] this group applied the optimised protocol to enrich the peptide from 211 fortified serum. The limit of detection from the optimised protocol was reported to 212 be about $600 \mathrm{pM}$. The elution protocol used $80 \%$ acetonitrile as elution solvent. 213 The MIP retained the targeted peptide more than the NIP, which nonetheless 214 does show non-specific binding. Importantly an example showed a much cleaner 215 chromatogram for the MIP compared with the NIP. Although a nonapeptide rather 
216 than a protein, this paper illustrates the potential of a method based on selective

217 SPE with a MIP followed by LC-MS for an important low abundance biomarker.

218 There are several other examples of polypeptide MIPs [30, 40, 42, 43]. Shinde et

219 al. 2012 [44] described how an SPE MIP format could distinguish between

220 sulpho- and phosphorylated peptides. Fractions were analysed by HPLC and

221 matrix assisted laser desorption ionisation (MALDI) to confirm the elution fraction

222 contents.

223

224 Qin et al. 2009 [45] showed the possibility of enriching lysozyme from aqueous 225 and biological samples - in this case egg white. $N$-(4-vinyl)-benzyl iminodiacetic 226 acid (VBIDA) was co-polymerized with $N$-isopropylacrylamide (NiPAm) and AAm 227 in the presence of copper $\left(\mathrm{Cu}^{2+}\right)$ ions. Greater adsorption capacity was shown for 228 the lysozyme template than for several other proteins (cytochrome $\mathrm{C}$ (CytC), 229 ribonuclease $A(R N a s e ~ A)$, ovalbumin, bovine haemoglobin $(B H b), B S A$, and 230 glucose oxidase). A gel electrophoresis figure showed enrichment of the 231 lysozyme from diluted egg white. There is growing interest in incorporating metal 232 ions (through complexation) to improve the binding affinity of MIP for a target 233 protein [46]. The electron donating effect of amino groups of the protein to the 234 metal centre offers an additional anchor point for the protein to dock within the 235 vicinity of the cavity.

237 Gao et al. 2010 [47] prepared a surface modified MIP to lysozyme using 238 methacrylic acid (MAA) as functional monomer and hydroxyethylmethacrylate 
239 (HEMA)/ N-vinylpyrrolidone (VNP) as cross-linked microspheres. Although

240 biological samples were not evaluated, dynamic binding curves clearly illustrated

241 the delayed elution of the lysozyme compared to bovine haemoglobin.

242

243 Gai et al. 2010 and 2011 [48, 49] prepared MIPs to BHb and lysozyme. The

244 lysozyme MIP was surface imprinted and showed greater selectivity for the

245 lysozyme compared with BHb, myoglobin, BSA, Trypsin inhibitor ( $\mathrm{TI}$ ) and CytC.

246 The BSA MIP similarly showed greater selectivity in adsorption experiments,

247 potentially applicable as a sample preparation/enrichment method. Non-specific

248 binding to NIP was also shown which could lessen the use of such a MIP for

249 accurate measurement.

250

251 Dan et al. 2013 [28] reported MIPs to ovalbumin using the polysaccharide

252 chitosan and acrylamide as monomers and described extensive optimisation of

253 synthesis. Selectivity was ascertained by comparing MIP rebinding with the non-

254 cognate proteins BSA, BHb and lysozyme. They also looked at surface

255 morphology using several techniques. Gels using chitosan and acrylic acid (AA)

256 and MAA showed the best potential but non-selective binding to NIP and

257 selectivity to other proteins still needs addressing. Biological samples were not

258 evaluated.

259

260 Wan et al. 2015 [50] showed how a polydopamine MIP surface imprinted on 261 nanoparticles could enrich lysozyme spiked diluted egg white samples. The MIP 
262 was compared to NIP and cross reactivity studies versus five proteins (RNase A, $263 \mathrm{BHb}, \mathrm{BSA}$, trypsin and $\mathrm{CytC}$ ) demonstrated preferential binding to the target 264 protein. Samples were analysed using MALDI-TOF.

266 Deng et al. 2011 [51] prepared a monolithic MIP to BSA using a freeze thawing 267 polymerisation method with acrylamide as the monomer. Both HPLC and SPE 268 demonstrated a greater retention for the BSA versus $\mathrm{Hb}$. A gel electrophoresis 269 plate showed a SPE extract enriched with the target protein compared to 270 carbonic anhydrase, lysozyme, BSA, and trypsin. The MIP column showed the 271 BSA, the NIP column showed none of the aforementioned proteins.

273 Lin et al. 2013 [52] described the selective extraction of horseradish peroxidise 274 (HRP) from spiked human serum samples. Dopamine was the functional 275 monomer used for MIP preparation. Although the paper was mainly concerned 276 with a monolithic HPLC column it also described the use of the MIP approach in 277 SPME format. It showed a gel electrophoresis plate with significantly enriched 278 HRP.

280 Namatozola et al. 2014 [53] used AAm to prepare MIPs for human serum 281 albumin (HSA) and IgG. Part of their article described the evaluation using SPE. 282 Comparison of MIP and NIP shows a slightly increased recovery in the elution 283 fraction for the imprinted protein particularly for the IgG. For both MIPs much of 
284 the protein was eluted in load and wash fractions suggesting very low selective 285 binding capacities within the MIP.

287 Solemani et al. 2012 [54] described the preparation of a BSA MIP under the 288 conditions normally used for small molecule analytes. They evaluated the MIP in 289 SPE format, optimising the flow rate, the effect of $\mathrm{pH}$, ionic strength, sample 290 volume and different ratios of methanol/acetonitrile on elution. After optimisation 291 with standard solutions, more challenging solutions such as serum, urine, whey 292 and milk were applied. MIPs were compared with NIPs for recovery. It should be 293 noted that elution fractions from the SPE columns were evaluated by UV-Vis 294 spectrophotometry not by chromatography or MS. The possibility of denaturation 295 of the BSA during MIP synthesis or the analytical protocol cannot be discounted 296 and could be evaluated by, for example, using circular dichroism spectroscopy to 297 assess the nature of the protein during and following the MIP production process $298[55,56]$.

300 Liu et al. 2014 [57] prepared MIPs for extraction of HSA using porcine serum 301 albumin as a dummy template with methacrylate monomers. The aim of this work 302 was to selectively extract high abundance protein that was not the analyte of 303 interest, thereby enhancing the detection limits of low abundance proteins of 304 interest. Much higher binding affinity for the desired protein was obtained 305 compared with $\beta$-lactoglobulin, CytC or ribonuclease B. The use of a dummy 306 template was common with small molecule SPE. It involved the use of a 
307 structural analogue of the target analyte to form the MIP. To date it is much less

308 common with macromolecules.

309

310 An example of virus imprinting was shown by Sykora et al. 2015 [58] where

311 preliminary results indicated the synthesis of surface MIPs to a Human Norovirus

312 strain. They pointed out some of the difficulties of this type of work. Quite apart

313 from the problem of biomolecule stability, the need to use large amounts of

314 pathogenic virus in the MIP synthesis stage restricts this type of work. This issue

315 was overcome by using a genetically modified virus-like particle as the template.

316 The paper showed a much larger binding to the MIP compared with the NIP.

317 Field emission scanning electron microscopy pictures were also shown as

318 evidence of MIP structure.

320 Comments

321 Sample preparation includes trying to isolate the analyte to improve detection

322 limits, especially if the analyte is at very low concentration when there is plenty of

323 sample. It can also include trying to remove matrix components that interfere

324 even if they do not give a direct signal to the detector, for example ion

325 suppression in MS.

326

327 In contrast to MIPs, antibodies are extensively used commercially especially in 328 clinical (bio) chemistry laboratories. There are examples where MIPs have been 329 shown to replace antibodies in clinical tests $[13,59]$. So their increasing use for 
330 selective extraction of macromolecules is anticipated. Whether it will be for

331 special applications or widespread depends on the reality of commercially

332 developing suitable products. The virtues of combining immunoaffinity sample

333 preparation with MS detection have been highlighted in a special issue of

334 Bioanalysis especially in the overview given by Ackerman [60]. The advantages

335 offered by biological antibodies will be potentially superseded if suitable MIPs can

336 be reliably produced. The attraction of specific analyte capture, trace enrichment

337 from a large volume and then release into a small volume of liquid compatible

338 with injection into an LC-MS is clear. The use of antibodies for this is increasing.

339 If this type of procedure could be achieved with MIPs this would be an even more

340 attractive approach.

342 With proteins and other large biomolecules analyte stability is a problem, so

343 aqueous based SPE protocols are essential. Several papers look at morphology

344 or cavity size, but to be of use to bioanalysts with real measurements to make

345 and defend this ultimately depends on how clean the samples are and the

346 reproducibility of results that is demanded by the end user. One of the drawbacks

347 with the use of MIPs has been the reality that they are not yet as specific as

348 biologically developed antibodies. Whereas $K_{d}$ values for antibody-antigen

349 interactions are of the order of $10^{-9} \mathrm{M}$, the majority of MIP-antigen interactions

350 are still at the $10^{-6}-10^{-7} \mathrm{M}$ range, However, recently Piletsky's group has

351 developed a technique for the mass production of nanosized MIPs (plastic

352 antibodies) reporting $\mathrm{K}_{d}$ values matching biological antibodies [61]. When used 
353 as reagents for SPE followed by a specific and sensitive end-step such as LC-

354 MS the lack of high affinity MIPs is less of a drawback. Potentially they can offer

355 enough selectivity in extraction to provide a clean enough sample for the

356 chromatography or other measurement. The reality that there are currently few

357 examples of this approach suggests it is worthy of more effort.

359 Peptides are not as challenging because they are more stable than proteins and 360 also less expensive in terms of requiring a relatively large amount of template.

361 Other similar approaches for selective extraction have also been developed. The 362 use of aptamers (short single stranded DNA or RNA molecules) has been 363 reviewed by $[62,63]$ including their use in SPE format. The importance of 364 measuring new therapeutic agents or small abundance protein biomarkers 365 means that the quest for improved methods of selective enrichment/clean-up will 366 continue. Other areas where MIPs may show promise include virus imprinting 367 [64-66] where preliminary experiments showed that tobacco mosaic virus could 368 be imprinted using polyallylamine.

370 Difficulties such as the need for a large amount of template for MIP synthesis,

371 reliable and complete template removal, minimisation of non-specific binding, a 372 reasonable shelf-life and commercial availability of quality controlled products 373 that are suitable for rebinding in aqueous solutions still need to be overcome. 374 Nonetheless the approach of selective (enough) extraction followed by HPLC-MS 
375 is an attractive proposition in bioanalysis. Hence, the development and validation

376 to regulatory authority guidelines of macromolecule MIPs is tentatively awaited.

377

\section{Conclusions}

379 Molecularly imprinted polymers offer an alternative approach to biological

380 antibodies for selective capture reagents in bioanalytical chemistry. Most of the

381 developments in MIPs have involved small molecules particularly drugs and

382 metabolites. Although several different applications have been proposed, none

383 have come into widespread routine use in laboratories. Use as selective sorbents

384 for SPE have been the most promising area. Even in this area, uptake has been

385 slow. This is in part due to the advent of techniques such as LC-MS seemingly

386 requiring less rigorous sample preparation requirements. It is also, in part,

387 caused by the nature of the technique. If you develop a product that is specific to

388 only one drug or class of drug - it is not going to attract a big market. However

389 generic protocols would be helpful here.

390

391 There is growing interest in accurate measurement of proteins and other

392 macromolecules or biological entities such as viruses. These are being

393 introduced as new drugs or being validated as biomarkers both for drug efficacy

394 and diagnostics. Not surprisingly, MIPs are being produced to macromolecules

395 and are now being evaluated for use in sensors and for sample preparation.

396 Selective extraction both for analytical and preparative purposes is worthy of 397 more research as there are few examples of macromolecule determination in 
398 biological samples. Methods proposed will need to be subject to the rigorous 399 validation protocols required by regulatory authorities, not just publication in 400 academic journals.

401

\section{Future Perspectives}

403 The determination of large molecules in biological fluids will continue to be an 404 area of growing importance. Problems with determining intact macromolecules 405 will present greater challenges than for small molecules not least due to their lack 406 of stability. Improvements in the preparation of macromolecular MIPs are 407 needed. This will facilitate investigations into the use of such selective reagents 408 for improved methods of sample preparation. These could then be utilised along 409 with methods such as LC-MS to provide accurate quantification at low 410 concentrations in biological fluids.

\section{Keywords}

413 Proteins, Macromolecules, Selective extraction, Molecular imprinted polymers, 414 Antibody mimics, Bioanalysis 


\section{Table 1 - Example of analytes imprinted within a varied mix of matrices and}

\section{7 monomer/cross-linker combinations.}

\begin{tabular}{|c|c|c|c|c|c|}
\hline Analyte & Matrix & Monomer & Cross-linker & Validation & Ref \\
\hline $\mathrm{BHb}$ & Aqueous buffers & AAm & MBAA & $\begin{array}{l}\text { MIP vs NIP } \\
\text { Selectivity vs BSA }\end{array}$ & [49] \\
\hline Lysozyme & $\begin{array}{l}\text { Aqueous and } \\
\text { diluted egg white }\end{array}$ & NiPAm/AAm & MBAA & $\begin{array}{l}\text { MIP vs NIP } \\
\text { Selectivity vs BSA, } \\
\text { Mb, BHb, TI, CytC }\end{array}$ & [48] \\
\hline Ovalbumin & $\begin{array}{l}\text { Aqueous } \\
\text { biological }\end{array}$ & $\begin{array}{l}\text { Chitosan/AA,AAm, } \\
\text { MAA }\end{array}$ & MBAA & $\begin{array}{l}\text { MIP vs NIP } \\
\text { Selectivity vs BSA, } \\
\text { BHb, lysozyme }\end{array}$ & [28] \\
\hline ProGRP & $\begin{array}{l}\text { Aqueous non- } \\
\text { biological }\end{array}$ & EAMA & DVB & $\begin{array}{l}\text { MIP vs NIP } \\
\text { Selectivity vs } 3 \\
\text { other poly peptides }\end{array}$ & {$[40,41]$} \\
\hline Lysozyme & Aqueous buffers & $\begin{array}{l}\text { VBDIA/ NiPAm/AAm } \\
\text { Plus Cu ions }\end{array}$ & MBAA & $\begin{array}{l}\text { MIP vs NIP } \\
\text { Selectivity vs } \\
\text { CytC, RNasaA, } \\
\text { OB, BSA, Hb, GOx }\end{array}$ & [45] \\
\hline Lysozyme & $\begin{array}{l}\text { Aqueous and } \\
\text { diluted egg white }\end{array}$ & Dopamine & Not reported & $\begin{array}{l}\text { MIP vs NIP and } \\
\text { selectivity vs CytC, } \\
\text { RNase A, BHb, } \\
\text { BSA, CytC }\end{array}$ & {$[50]$} \\
\hline HPR & $\begin{array}{l}\text { Spiked human } \\
\text { serum }\end{array}$ & Dopamine & PETA & $\begin{array}{l}\text { HSA, IgG, Trf and } \\
\text { other } \\
\text { proteins }\end{array}$ & {$[52]$} \\
\hline HSA, IgG & Aqueous buffers & AAm & MBAA & MIP vs NIP & [53] \\
\hline BSA & Aqueous buffers & AAm & MBAA & $\begin{array}{l}\text { MIP vs NIP and } \\
\text { selectivity vs CA, } \\
\text { lysozyme, BSA, } \\
\text { and trypsin }\end{array}$ & {$[51]$} \\
\hline BSA & $\begin{array}{l}\text { Aqueous buffers, } \\
\text { serum, urine }\end{array}$ & 2VP & EGDMA & MIP vs NIP & {$[54]$} \\
\hline
\end{tabular}

Acrylamide (AAm); Acrylic acid (AA); Methylacrylic acid (MAA); N-(2-Aminoethyl methacrylamide hydrochloride (EAMA); N-isopropylacrylamide (NiPAm); Divinyl benzene (DVB); N,N-methylenebisacrylamide (MBAA); N-(4-vinyl)benzyl iminodiacetic acid (VBIDA); 2-vinylpyridine (2VP); Cytochrome C (CytC); Bovine haemoglobin (BHb); Bovine serum albumin (BSA); Myoglobin (Mb); Trypsin inhibitor (TI); Glucose oxidase (GOx); Carbonic Anhydrase (CA); Ovalbumin (OB); Pentaerythritol triacrylate (PETA); Horseradish peroxidase (HPR); Transferrin (Trf); Ribonuclease A (RNase A); Ethylene glycol dimethylacrylate (EGDMA). 


\section{Executive Summary}

\section{Background}

- The measurement of drugs, metabolites and endogenous compounds is a very challenging area for Analytical Chemists. The most common methods involve some form of extraction to give sample clean up and preconcentration. This is then followed by injection into a gas or liquid chromatograph and measurement using a variety of detectors but most commonly nowadays mass spectrometry.

- As demands for better sensitivity are a challenge methods of selective extraction have been explored. One of the most attractive of these has been the use of immobilised antibodies to selectively extract drugs and metabolites using solid phase extraction.

\section{Molecularly Imprinted Polymers}

- MIPs are synthetic polymers formed around a template molecule (the analyte). These are then used as reagents to selectively rebind the analyte during sample preparation. They are much cheaper than biological antibodies and are more stable.

- There are many literature applications using MIPs to extract small molecular mass drugs and metabolites but they are not in common use in industrial laboratories.

\section{MIPs for extraction/enrichment of macromolecules}


- With the development of macromolecules as candidate drugs and biomarkers there has been increased interest in developing selective extraction to large molecules.

- The use of soft gels, where the MIPs are formed in aqueous solutions is much more applicable to biomolecules which are generally not stable in

448 Examples of extraction/enrichment of macromolecules using MIPs

- Examples of selective binding of a number of macromolecules are given. These include peptides and polypeptides, lysozyme, bovine haemoglobin, bovine serum albumin, ovalbumin, horseradish peroxidise, human serum albumin, and viruses.

\section{Comments}

- The combination of selective extraction along with HPLC-MS to measure

457 - However there are as yet few examples where this has been achieved with MIPs as opposed to biological antibodies.

- There are some questions as to whether or not a MIP effect is as selective as desired.

\section{Conclusions}


- Use of selective extraction is an area likely to grow as more macromolecular drug candidates and biomarkers are developed.

465 
467 1. Soltani S, Jouyban A. Biological sample preparation: attempts on productivity 468 increasing in bioanalysis. Bioanalysis. 6(12), 1691-1710 (2014).

469 2. Stevenson D. Bioanalysis through the ages. Bioanalysis. 3(24), 2681-2683 470 (2011).

471 3. Stevenson D. Immuno-affinity solid-phase extraction. J. Chromatogr. B. 472 745(1), 39-48 (2000).

473 4. Hjerten S, Liao JL, Nakazato K, Wang Y, Zamaratskaia G, Zhang HX. Gels 474 mimicking antibodies in their selective recognition of proteins. Chromatographia. 475 44(5-6), 227-234 (1997).

476 5. Bowen JL, Manesiotis P, Allender CJ. Twenty years since 'antibody mimics' by 477 molecular imprinting were first proposed: A critical perspective. Molecular 478 Imprinting. 1, 35-40 (2013).

479 6. EL-Sharif HF, Stevenson D, Warriner K, Reddy SM. Hydrogel-Based 480 Molecularly Imprinted Polymers for Biological Detection. In: Advanced Synthetic 481 Materials in Detection Science. Reddy SM (Ed.), The Royal Society of Chemistry, 482 UK, 75-115 (2014).

483 7. Beltran A, Borrull F, Cormack PAG, Marce RM. Molecularly-imprinted 484 polymers: useful sorbents for selective extractions. TrAC. 29(11), 1363-1375 485 (2010).

486 8. El-Sharif HF, Phan QT, Reddy SM. Enhanced selectivity of hydrogel-based 487 molecularly imprinted polymers (HydroMIPs) following buffer conditioning. Anal. 488 Chim. Acta. 809(0), 155-161 (2014).

489 9. EL-Sharif H, Hawkins DM, Stevenson D, Reddy SM. Determination of protein 490 binding affinities within hydrogel-based molecularly imprinted polymers 491 (HydroMIPs). Phys. Chem. Chem. Phys. 16(29), 15483-15489 (2014).

492 10. Stevenson D. Molecular imprinted polymers for solid-phase extraction. TrAC. 493 18(3), 154-158 (1999).

494 11. Martin-Esteban A. Molecularly-imprinted polymers as a versatile, highly 495 selective tool in sample preparation. TrAC. 45, 169-181 (2013).

496 12. Moreno-Gonzalez D, Lara FJ, Gamiz-Gracia L, Garcia-Campana AM. 497 Molecularly imprinted polymer as in-line concentrator in capillary electrophoresis 
498 coupled with mass spectrometry for the determination of quinolones in bovine 499 milk samples. J. Chromatogr. A. 1360, 1-8 (2014).

500 13. Chianella I, Guerreiro A, Moczko E et al. Direct Replacement of Antibodies 501 with Molecularly Imprinted Polymer Nanoparticles in ELISA-Development of a 502 Novel Assay for Vancomycin. Anal. Chem. 85(17), 8462-8468 (2013).

503 14. Fatoni A, Numnuam A, Kanatharana P, Limbut W, Thavarungkul P. A novel 504 molecularly imprinted chitosan-acrylamide, graphene, ferrocene composite 505 cryogel biosensor used to detect microalbumin. Analyst. 139(23), 6160-6167 506 (2014).

507 15. Saridakis E, Khurshid S, Govada L et al. Protein crystallization facilitated by 508 molecularly imprinted polymers. PNAS. 108(27), 11081-11086 (2011).

509 16. Arabi M, Ostovan A, Ghaedi M, Purkait MK. Novel strategy for synthesis of 510 magnetic dummy molecularly imprinted nanoparticles based on functionalized 511 silica as an efficient sorbent for the determination of acrylamide in potato chips: 512 Optimization by experimental design methodology. 154, 526-532 (2016).

513 17. Arabi M, Ghaedi M, Ostovan A, Tashkhourian J, Asadallahzadeh $\mathrm{H}$. 514 Synthesis and application of molecularly imprinted nanoparticles combined 515 ultrasonic assisted for highly selective solid phase extraction trace amount of 516 celecoxib from human plasma samples using design expert (DXB) software. 517 TalantaUltrason. Sonochem. 33, 67-76 (2016).

518 18. Asfaram A, Ghaedi M, Dashtian K. Rapid ultrasound-assisted magnetic 519 microextraction of gallic acid from urine, plasma and water samples by HKUST-1520 MOF-Fe3O4-GA-MIP-NPs: UV-vis detection and optimization study. Ultrason. 521 Sonochem. 34, 561-570 (2017).

522 19. Asfaram A, Ghaedi M, Dashtian K. Ultrasound assisted combined molecularly 523 imprinted polymer for selective extraction of nicotinamide in human urine and 524 milk samples: Spectrophotometric determination and optimization study. 525 Ultrason. Sonochem. 34, 640-650 (2017).

526 20. Arabi M, Ghaedi M, Ostovan A. Development of dummy molecularly 527 imprinted based on functionalized silica nanoparticles for determination of 528 acrylamide in processed food by matrix solid phase dispersion. Food Chem. 210, 529 78-84 (2016).

530 21. Cai Q, Yang Z, Chen N, Zhou X, Hong J. Selective capture and rapid 531 identification of Panax notoginseng metabolites in rat faeces by the integration of 532 magnetic molecularly imprinted polymers and high-performance liquid 533 chromatography coupled with orbitrap mass spectrometry. J. Chromatogr. A. 534 1455, 65-73 (2016). 
22. Ding X, Heiden PA. Recent Developments in Molecularly Imprinted Nanoparticles by Surface Imprinting Techniques. Macromol. Mater. Eng. 299(3), 268-282 (2014).

23. Cheong WJ, Yang SH, Ali F. Molecular imprinted polymers for separation science: A review of reviews. J. Sep. Sci. 36(3), 609-628 (2013).

24. Li S, Cao S, Whitcombe MJ, Piletsky SA. Size matters: Challenges in imprinting macromolecules. Progress in Polymer Science. 39(1), 145-163 (2014).

25. Hawkins DM, Stevenson D, Reddy SM. Investigation of protein imprinting in hydrogel-based molecularly imprinted polymers (HydroMIPs). Anal. Chim. Acta. 542(1), 61-65 (2005).

26. Hawkins DM, Ellis EA, Stevenson D, Holzenburg A, Reddy SM. Novel critical point drying (CPD) based preparation and transmission electron microscopy (TEM) imaging of protein specific molecularly imprinted polymers (HydroMIPs). $J$. Mater. Sci. 42(22), 9465-9468 (2007).

27. Hawkins DM, Trache A, Ellis EA et al. Quantification and confocal imaging of protein specific molecularly imprinted polymers. Biomacromolecules. 7(9), 25602564 (2006).

28. Dan R, Wang Y, Du L et al. The synthesis of molecular imprinted chitosangels copolymerized with multiform functional monomers at three different temperatures and the recognition for the template ovalbumin. Analyst. 138(12), 3433-3443 (2013).

29. Hu J, Mao X, Cao S, Yuan X. Recognition of Proteins and Peptides: Rational Development of Molecular Imprinting Technology. Polymer Science Series a. 52(3), 328-339 (2010).

30. Bossi A, Bonini F, Turner APF, Piletsky SA. Molecularly imprinted polymers for the recognition of proteins: The state of the art. Biosens. Bioelectron. 22(6), 1131-1137 (2007).

31. Whitcombe MJ, Chianella I, Larcombe $L$ et al. The rational development of molecularly imprinted polymer-based sensors for protein detection. Chem. Soc. Rev. 40(3), 1547-1571 (2011).

32. Chen L, Xu S, Li J. Recent advances in molecular imprinting technology: current status, challenges and highlighted applications. Chem. Soc. Rev. 40(5), 2922-2942 (2011). 
33. Chen $\mathrm{H}$, Zhang $\mathrm{Z}$, Cai $\mathrm{R}$ et al. Molecularly imprinted electrochemical sensor based on amine group modified graphene covalently linked electrode for 4nonylphenol detection. Talanta. 115(0), 222-227 (2013).

34. Augusto F, Hantao LW, Mogollon NGS, Braga SCGN. New materials and trends in sorbents for solid-phase extraction. TrAC. 43, 14-23 (2013).

35. Hu Y, Pan J, Zhang K, Lian H, Li G. Novel applications of molecularlyimprinted polymers in sample preparation. TrAC. 43, 37-52 (2013).

36. Schirhagl R. Bioapplications for Molecularly Imprinted Polymers. Anal. Chem. 86(1), 250-261 (2014).

37. Wen L, Tan X, Sun Q, Svec F, Lv Y. "Smart" molecularly imprinted monoliths for the selective capture and easy release of proteins. J. Sep. Sci, 1-7 (2016).

38. Verheyen E, Schillemans JP, van Wijk M, Demeniex M, Hennink WE, van Nostrum CF. Challenges for the effective molecular imprinting of proteins. Biomaterials. 32(11), 3008-3020 (2011).

39. Mike Whitcombe w. MIPdatabase.com. MIPdatabase.com. 2016 (2015).

40. Qader AA, Urraca J, Torsetnes SB, Tonnesen F, Reubsaet L, Sellergren B. Peptide imprinted receptors for the determination of the small cell lung cancer associated biomarker progastrin releasing peptide. J. Chromatogr. A. 1370, 5662 (2014).

*41. Rossetti C, Qader AA, Halvorsen TG, Sellergren B, Reubsaet L. AntibodyFree Biomarker Determination: Exploring Molecularly Imprinted Polymers for ProGastrin Releasing Peptide. Anal. Chem. 86(24), 12291-12298 (2014).

42. Helling S, Shinde S, Brosseron F et al. Ultratrace Enrichment of Tyrosine Phosphorylated Peptides on an Imprinted Polymer. Anal. Chem. 83(5), 18621865 (2011).

43. Li Q, Shen F, Zhang X et al. One-pot synthesis of phenylphosphonic acid imprinted polymers for tyrosine phosphopeptides recognition in aqueous phase. Anal. Chim. Acta. 795, 82-87 (2013).

44. Shinde S, Bunschoten A, Kruijtzer JAW, Liskamp RMJ, Sellergren B. Imprinted Polymers Displaying High Affinity for Sulfated Protein Fragments. Angewandte Chemie-International Edition. 51(33), 8326-8329 (2012).

45. Qin L, He X, Zhang W, Li W, Zhang Y. Macroporous Thermosensitive Imprinted Hydrogel for Recognition of Protein by Metal Coordinate Interaction. Anal. Chem. 81(17), 7206-7216 (2009). 
602 46. EL-Sharif HF, Yapati H, Kalluru S, Reddy SM. Highly selective BSA imprinted 603 polyacrylamide hydrogels facilitated by a metal-coding MIP approach. Acta 604 Biomaterialia. 28(0), 121-127 (2015).

605 47. Gao B, Fu H, Li Y, Du R. Preparation of surface molecularly imprinted 606 polymeric microspheres and their recognition property for basic protein lysozyme. 607 J. Chromatogr. B. 878(21), 1731-1738 (2010).

608 48. Gai Q, Qu F, Zhang Y. The Preparation of BHb-Molecularly Imprinted Gel 609 Polymers and Its Selectivity Comparison to BHb and BSA. Sep. Sci. Technol. 610 45(16), 2394-2399 (2010).

611 49. Gai $Q$, Qu F, Zhang T, Zhang Y. The preparation of bovine serum albumin 612 surface-imprinted superparamagnetic polymer with the assistance of basic 613 functional monomer and its application for protein separation. J. Chromatogr. A. 614 1218(22), 3489-3495 (2011).

615 50. Wan W, Han Q, Zhang X, Xie Y, Sun J, Ding M. Selective enrichment of 616 proteins for MALDI-TOF MS analysis based on molecular imprinting. Chem. 617 Commun. 51(17), 3541-3544 (2015).

618 *51. Deng QL, Li YL, Zhang LH, Zhang YK. Molecularly imprinted macroporous 619 monolithic materials for protein recognition. Chinese Chemical Letters. 22(11), 620 1351-1354 (2011).

621 52. Lin Z, Wang J, Tan $X$ et al. Preparation of boronate-functionalized 622 molecularly imprinted monolithic column with polydopamine coating for 623 glycoprotein recognition and enrichment. J. Chromatogr. A. 1319, 141-147 624 (2013).

625 53. Nematollahzadeh A, Lindemann P, Sun W, Stute J, Luetkemeyer D, 626 Sellergren B. Robust and selective nano cavities for protein separation: An 627 interpenetrating polymer network modified hierarchically protein imprinted 628 hydrogel. J. Chromatogr. A. 1345, 154-163 (2014).

629 54. Soleimani M, Ghaderi S, Afshar MG, Soleimani S. Synthesis of molecularly 630 imprinted polymer as a sorbent for solid phase extraction of bovine albumin from 631 whey, milk, urine and serum. Microchemical Journal. 100, 1-7 (2012).

632 55. Kryscio DR, Fleming MQ, Peppas NA. Conformational studies of common 633 protein templates in macromolecularly imprinted polymers. Biomed. 634 Microdevices. 14(4), 679-687 (2012).

635 56. Kryscio DR, Fleming MQ, Peppas NA. Protein Conformational Studies for 636 Macromolecularly Imprinted Polymers. Macromolecular Bioscience. 12(8), 1137$6371144(2012)$. 
638 57. Liu J, Deng Q, Tao D et al. Preparation of protein imprinted materials by 639 hierarchical imprinting techniques and application in selective depletion of 640 albumin from human serum. Scientific Reports. 4, 5487 (2014).

$641{ }^{*} 58$. Sykora S, Cumbo A, Belliot G et al. Virus-like particles as virus substitutes to 642 design artificial virus-recognition nanomaterials. Chem. Commun. 51(12), 22566432258 (2015).

644 59. Bedwell TS, Whitcombe MJ. Analytical applications of MIPs in diagnostic 645 assays: future perspectives. Anal. Bioanal. Chem. 408(7), 1735-1751 (2016).

646 60. Ackermann BL. Immunoaffinity MS: adding increased value through hybrid 647 methods. Bioanalysis. 8(15), 1535-1537 (2016).

648 61. Canfarotta F, Poma A, Guerreiro A, Piletsky S. Solid-phase synthesis of 649 molecularly imprinted nanoparticles. Nat. Protoc. 11(3), 443-455 (2016).

650 62. Du F, Alam MN, Pawliszyn J. Aptamer-functionalized solid phase 651 microextraction-liquid chromatography/tandem mass spectrometry for selective 652 enrichment and determination of thrombin. Anal. Chim. Acta. 845, 45-52 (2014).

653 63. Pichon V, Brothier F, Combes A. Aptamer-based-sorbents for sample 654 treatment-a review. Anal. Bioanal. Chem. 407(3), 681-698 (2015).

655 64. Bolisay LD, Culver JN, Kofinas P. Molecularly imprinted polymers for tobacco 656 mosaic virus recognition. Biomaterials. 27(22), 4165-4168 (2006).

657 65. Bolisay LD, Culver JN, Kofinas P. Optimization of virus imprinting methods to 658 improve selectivity and reduce nonspecific binding. Biomacromolecules. 8(12), 659 3893-3899 (2007).

660 66. Bolisay LD, Kofinas P. Imprinted Polymer Hydrogels for the Separation of 661 Viruses. Macromol. symp. 291-292, 302-306 (2010). 
663

664 Highlights

66541 This paper shows a peptide MIP spiked into serum

66651 This paper shows an enriched SPE trace on gel electrophoresis

66758 This paper shows the possibility for virus imprinting.

668 